\title{
APLICACIÓN DE LA ORDEN EHA/3360/2010 SOBRE ASPECTOS CONTABLES DE LAS SOCIEDADES COOPERATIVAS: EFECTOS EN LOS FONDOS PROPIOS DE LAS COOPERATIVAS DE LA COMUNIDAD AUTÓNOMA DEL PAÍS VASCO
}

\author{
POR \\ Miguel Ángel ZUBIAURRE ARTOLA ${ }^{1}$ \\ Lorea ANDICOECHEA ARONDO ${ }^{2}$ \\ Ainhoa SAITUA IRIBAR ${ }^{3}$
}

\section{RESUMEN}

El objetivo de este trabajo es estudiar el impacto que ha supuesto sobre los fondos propios de las cooperativas de trabajo asociado de la Comunidad Autónoma del País Vasco la aplicación del nuevo marco normativo contable, consecuencia de la entrada en vigor de la Orden EHA/3360/2010, Normas sobre aspectos contables de las sociedades cooperativas. Por un lado, se analizan las diferentes medidas acordadas en las asambleas de las cooperativas a fin de proteger la consideración como patrimonio neto del capital cooperativo y otros fondos recuperables por los socios en caso de baja. Por otro lado, se ha estudiado el efecto que el cambio normativo ha tenido sobre las variables centrales del patrimonio neto y sobre los ratios financieros de solvencia de este tipo de sociedades. Para ello se analizan tanto cooperativas de trabajo asociado de más de 50 empleados, como las cooperativas de enseñanza de mayor tamaño y el caso específico de Eroski, por ser uno de los más relevantes socialmente en el ámbito de la CAPV. La aplicación del cambio normativo no ha tenido un impacto relevante sobre los fondos propios de la mayoría de las cooperativas de trabajo

\footnotetext{
1 Profesor Titular de la Universidad del País Vasco (UPV/EHU). Dirección de correo electrónico: ma.zubiaurre@ehu.es

2 Profesora Titular de la Universidad del País Vasco (UPV/EHU). Dirección de correo electrónico: 1.andicoechea@ehu.es

${ }^{3}$ Profesora Titular de Escuela Universitaria del País Vasco (UPV/EHU). Dirección de correo electrónico: ainhoa.saitua@ehu.es
}

REVESCO No 118 - Segundo Cuatrimestre 2015 - ISSN: 1885-8031 - www.ucm.es/info/revesco

http://dx.doi.org/10.5209/rev_REVE.2015.n118.49061

Fecha de recepción: 14/04/2014

Fecha de aceptación: 26/11/2014 
asociado con más de 50 empleados de Euskadi, fundamentalmente, porque las cooperativas han optado por cambiar sus condiciones estatutarias a fin de protegerse del efecto de la aplicación del nuevo marco normativo contable. Sin embargo, hemos constatado que las cooperativas que no han adaptado su marco estatutario a las exigencias de la nueva regulación se han visto forzadas a realizar reclasificaciones de fondos propios a pasivo, que han tenido un impacto patrimonial muy relevante.

Palabras clave: cooperativas de trabajo asociado, regulación contable, armonización internacional, patrimonio neto, capital, solvencia.

Claves Econlit: P130, M410, M480.

\title{
APPLICATION OF ORDER EHA/3360/2010 OVER ACCOUNTING ISSUES ON COOPERATIVE SOCIETIES: EFFECTS ON OWN FUNDS IN COOPERATIVES OF THE BASQUE COUNTRY
}

\begin{abstract}
The aim of this work is to study the effects that the implementation of a new accounting regulatory framework, which involved the application of the IAS 32, has caused on the equity of worker cooperatives in the Basque Country. On the one hand, we have analyzed different decisions that cooperatives have adopted in their assemblies to protect the consideration as equity of members' shares and others funds that are recoverable by members when they leave the cooperative. Furthermore, we have studied the effect that the accounting policy change has on the core variables of equity and in financial solvency ratios of these companies. According to our results, the accounting policy change did not have a significant impact on the equity of most worker cooperatives of Basque Country, mainly due to the fact, they have opted to change their statutory rules in order to protect cooperative's equity from the new accounting framework. However, we have found that the cooperatives have not adapted their statutes have been forced to make reclassifications from equity to liability, which have entailed a very significant impact on equity.
\end{abstract}

Keywords: worker cooperatives, accounting regulation, international harmonization, equity, capital, solvency. 


\section{INTRODUCCIÓN}

Recientemente las entidades cooperativas españolas se han visto sometidas a un nuevo marco normativo contable aprobado por la Orden EHA/3360/2010, de 21 de diciembre, por la que se aprueban las Normas sobre aspectos contables de las sociedades cooperativas. Este hecho ha supuesto la aplicación plena de las normas internacionales de contabilidad a las cooperativas dando por concluido el periodo transitorio abierto tras la aprobación del PGC del 2007. La aprobación de la nueva norma de contabilidad de cooperativas ha implicado, esencialmente, la asunción de la norma internacional de contabilidad NIC 32 Instrumentos Financieros: Presentación (IASB, 2011), así como de la interpretación de norma CINIIF 2, Aportaciones de Socios de Entidades Cooperativas e Instrumentos Similares (IASB, 2004), única normativa internacional específica para contabilidad de cooperativas. Previamente, tan solo las entidades financieras cooperativas y las cooperativas con emisión de instrumentos financieros cotizados se habían visto sometidas a dicha legislación. La aprobación de la Orden EHA 3360/2010 ha supuesto, por tanto, la aplicación a todas las cooperativas españolas de la citada interpretación de norma internacional.

Hemos de tener presente que la solución planteada por la NIC 32 y por su norma de interpretación CINIIF2 se ha mostrado insatisfactoria y ha sido objeto de debate a nivel internacional en el marco de diferentes documentos de discusión, tanto del FASB (2007) y del IASB (2008), como en proyectos conjuntos de ambos organismos. Algunas de las soluciones y decisiones provisionales incluyen la consideración como neto de los instrumentos únicamente reembolsables en casos de "retirement", concepto que los propios reguladores hacen extensible al cese como miembro de la cooperativa. En el presente, el IASB continúa con el debate en el marco del proyecto de discusión del marco conceptual tratando de definir los elementos de los estados financieros. En particular, resulta de especial relevancia el borrador de discusión Elements of financial statements: definition of equity and distinction between liabilities and equity instruments (IASB, 2013). Este borrador defiende ofrecer mayor información con relación a las posibles reclamaciones futuras sobre las partidas del neto, a la vez que propone el criterio "pure cash approach" para la distinción entre neto y pasivo. En los comentarios aportados se muestra partidario de buscar solución, como neto patrimonial, a instrumentos con opción de venta asimilables al caso de las aportaciones al capital de la cooperativa. 
Las normas internacionales de contabilidad, pensadas y desarrolladas para ser implantadas en sociedades de capital, presentan dificultades al ser aplicadas a entidades cooperativas donde se rompen algunas de las premisas básicas del funcionamiento de las sociedades de capital (López-Espinosa, Maddocks y Polo-Garrido, 2012; Andicoechea y Zubiaurre, 2012). La aplicación indiscriminada de una normativa contable basada en principios orientados para su aplicación en las sociedades de capital, presenta dificultades en entornos específicos como es el de las cooperativas. En el caso español, la entrada en vigor de la nueva norma ha supuesto una amenaza para las entidades cooperativas.

La amenaza más clara consistía en la pérdida de la condición de patrimonio neto de las aportaciones del capital de los socios de las cooperativas. La interpretación de uno de los principios básicos del modelo cooperativo asociado a la adhesión voluntaria y abierta de los socios, suponía para éste el derecho al rescate de los capitales aportados ante su decisión de abandono de la cooperativa. Bajo la interpretación de la normativa internacional, la incapacidad de los órganos de la cooperativa para evitar la salida de las aportaciones de capital ante la baja de los socios implica la calificación del capital como pasivo desde el instante de su aportación. Esta amenaza se extiende a otras partidas del neto, como son las reservas rescatables en caso de baja. Esta última opción, aún siendo limitada en el modelo cooperativo español, podría afectar a las reservas voluntarias calificadas como repartibles y otros fondos que en base a la legislación autonómica particular, podría rescatar el socio en el momento de su baja.

La aportación de capital de los socios ha sido tradicionalmente el componente central del neto patrimonial de las entidades cooperativas. Por tanto, su reclasificación como pasivo suponía un serio riesgo a la imagen de solvencia que ofrecerían las cuentas anuales de estas entidades. Un mero cambio normativo, sin mayor recorrido en la operativa y funcionamiento real de las cooperativas, podía suponer un cambio drástico en la imagen financiera derivada de la información contenida en las cuentas anuales. Este proceso se traduciría en el empeoramiento generalizado de los ratios financieros de solvencia más utilizados por analistas y entidades de crédito a la hora de evaluar la capacidad de hacer frente a las deudas contraídas. En un entorno de enorme estrechez del crédito como el actual, esta circunstancia implicaba un claro riesgo sobre la capacidad de obtener financiación ajena así como, en su caso, sobre el coste de dicha financiación. 
Con estos antecedentes y con el objetivo de evitar la reclasificación del capital cooperativo como pasivo como consecuencia de la aplicación de la nueva normativa contable, la Ley 16/2007, de 4 de julio, introdujo una serie de modificaciones en la Ley 27/1999 de Cooperativas, permitiendo que la Asamblea de la cooperativa modificase los estatutos, otorgando al Consejo Rector la facultad de rehusar incondicionalmente el reembolso de las aportaciones.

La Ley, aplicable a las cooperativas de competencia estatal, tuvo antecedentes similares en la legislación aplicable en las Comunidad Autónoma del País Vasco y Comunidad Foral de Navarra. Esta modificación legal, que se realizó con posterioridad en el resto de legislaciones autonómicas, abrió la posibilidad de mantener el capital cooperativo como fondo propio, siempre y cuando se otorgase al Consejo Rector o a la Asamblea General la facultad de rehusar incondicionalmente el derecho de rescate del capital en el caso de baja del socio.

Lógicamente, cada cooperativa deberá previamente decidir en asamblea si acuerda configurar la aportación del capital bajo esta nueva opción legal, o por el contrario, continúa con los planteamientos tradicionales en los que el socio mantiene el derecho de rescate incondicional. Si en asamblea se acuerda la reclasificación del capital a esta nueva condición, la cooperativa podrá mantener el capital como patrimonio neto. En este sentido, no debemos olvidar que el coste de la adaptación supone modificar un principio tradicional de la configuración de la relación socio-cooperativa.

En el caso particular de las cooperativas de trabajo asociado, la incorporación del socio a la cooperativa tiene como finalidad última establecer una relación de trabajo, siendo la aportación del capital un requisito necesario para la consecución del objetivo fundamental, la relación de trabajo. De este modo la asociación del socio con la cooperativa se mantendrá indisoluble durante la trayectoria laboral del socio en la cooperativa. La baja del socio será obligada cuando concluya la relación de trabajo, es decir, en caso de jubilación, incapacidad o mortis causa. Es el cese de la relación de trabajo el que hace surgir el derecho del socio al rescate del capital.

En este entorno de cambio normativo es de destacar la respuesta del Instituto de Contabilidad Auditoría de Cuentas (ICAC, 2011) con relación a la calificación contable del capital social exigible en caso de jubilación o incapacidad. En dicha consulta el ICAC 
manifiesta que, dada la especialidad que caracteriza a la cooperativas como "sociedades de personas", cuando dicha actividad no pueda seguir desarrollándose por imposición legal, como sucede en los supuestos de incapacidad y jubilación, se considera que el derecho de reembolso no califica la aportación como un pasivo si dichas circunstancias impiden la continuidad de la actividad cooperativizada, como pudiera ser el caso de las cooperativas de trabajo asociado.

Esta opinión, a nuestro juicio acertada, está en la línea de las manifestaciones y decisiones provisionales formuladas en torno al proyecto de discusión conjunto del IASB y FASB "Financial Instruments with Characteristics of Equity", paralizado en 2010 pero reactivado en 2012, aunque como proyecto solo del IASB y supeditado al proyecto de marco conceptual (IASB, 2010). No obstante, la opinión manifestada en la consulta no se desprende, a nuestro juicio, del contenido de la Orden EHA/3360/2010, con la que, a nuestro entender, podría entrar en contradicción.

En este trabajo estudiamos el proceso de adaptación de las cooperativas ante el nuevo marco normativo, así como las principales consecuencias que ha supuesto sobre variables del patrimonio neto en términos cuantitativos. De este modo, los objetivos del trabajo son, por un lado, analizar cuál ha sido la reacción de las cooperativas ante la amenaza que implicaba la reclasificación de su capital como pasivo. Para ello, se han estudiado las diferentes medidas acordadas en las asambleas de las cooperativas a fin de proteger la consideración como neto del capital cooperativo y de los otros fondos recuperables por los socios de la cooperativa.

Por otro lado, el trabajo evalúa el impacto real que la aplicación de la Orden EHA/3360/2010 ha supuesto sobre el importe de las variables centrales del patrimonio neto y los ratios financieros de solvencia estudiados en la literatura (Marí-Vidal, 2006; Vargas, 2007 y 2011; Fernández, 2007; Arias y Montegut, 2012). Con este fin se ha analizado el impacto del cambio normativo sobre las siguientes variables: capital cooperativo, reservas acumuladas, fondos capitalizados, fondos propios totales y ratio de solvencia global.

\section{METODOLOGÍA}

La investigación se centra en las cooperativas de trabajo asociado que constituyen el tipo de cooperativas de mayor impacto económico en el País Vasco, tanto por volumen de operaciones como por número de trabajadores. Además, el caso de las cooperativas de trabajo asociado adquiere especial relevancia a raíz de la consulta publicada por el Instituto de 
Contabilidad y Auditoría de Cuentas (ICAC, 2011). En ella se menciona expresamente a las cooperativas de trabajo asociado como ejemplo particular de reclasificación por la vinculación legal que se produce entre reembolso de capital y cese de la actividad cooperativizada. Por lo tanto, es en estas cooperativas donde los casos de bajas de socios por supuestos de incapacidad y jubilación adquieren mayor interés.

En este trabajo hemos analizado la información financiera presentada por las empresas cooperativas al cierre del ejercicio a 31/12/2011, en cuentas anuales individuales. Se trata del primer cierre en el que resulta de obligado cumplimiento la aplicación de la nueva norma de contabilidad de cooperativas y, por tanto, la primera ocasión para estudiar su impacto. Las cuentas anuales presentadas en cierres a fechas previas a lo largo del 2011 no resultaban de interés dado que no recogían el efecto de la aplicación de la nueva norma, al tratarse de ejercicios económicos iniciados con anterioridad al 1 de enero del 2011, por lo que han sido descartadas.

Con el objetivo de estudiar las cooperativas que presentan mayor calidad en la información revelada, se consideró oportuno centrar el estudio en las cooperativas sometidas a procesos de auditoría externa. Sin embargo, ni el Registro de Cooperativas de Euskadi ni la Federación de Cooperativas de Trabajo Asociado, Enseñanza y Crédito del País Vasco (ERKIDE) permitían identificar dicha relación de cooperativas. Como alternativa, basándonos en los registros disponibles en ERKIDE, se optó por limitar las cooperativas objeto de estudio en función del número de trabajadores. Así, se han tomado las cooperativas de trabajo asociado de más de 50 trabajadores, que tengan domicilio social en la comunidad autónoma del País Vasco. De las 809 cooperativas de trabajo asociado inscritas en ERKIDE ${ }^{4}$ cumplían este criterio 92 empresas.

Identificadas las empresas objetivo del estudio, se ha tenido acceso a las cuentas anuales de 71 cooperativas de trabajo asociado. Analizada la documentación recibida, se identificó que tres de ellas no cumplían los parámetros establecidos (dos empresas contaban con menos de 50 trabajadores y una de ellas se había transformado como cooperativa a lo largo del 2011, por lo que ofrecía exclusivamente información del año 2011).

\footnotetext{
${ }^{4}$ En abril del 2013 Erkide contaba con 891 cooperativas inscritas, de las que 809 son cooperativas de trabajo asociado, 80 de enseñanza y 2 de crédito. Entre las cooperativas de trabajo asociado, 456 son empresas del sector servicios y transporte, 241 son industriales y 112 del sector construcción.
} 
Tabla 1: Número de cuentas anuales de cooperativas de trabajo asociado, solicitadas y analizadas

\begin{tabular}{lc}
\hline \multicolumn{2}{c}{$\begin{array}{c}\text { Cooperativas de Trabajo Asociado del País Vasco } \\
\text { de más } 50 \text { trabajadores inscritas en ERKIDE }\end{array}$} \\
\hline Cuentas Anuales del ejercicio 2011 solicitadas & 92 \\
Depositadas a fecha abril de 2013 & 71 \\
Rechazadas & 3 \\
\hline Estudiadas & $\mathbf{6 8}$
\end{tabular}

Fuente: Elaboración propia

Como caso de contrate se decidió estudiar las cooperativas de enseñanza, entre las que se han identificado 53 cooperativas inscritas en ERKIDE. Aplicando los criterios de tamaño se solicitaron cuentas de 17 cooperativas de enseñanza. Sin embargo, 14 de las cooperativas solicitadas cerraban el ejercicio en agosto, de acuerdo con el curso escolar, por tanto, no contaban con información de interés para el estudio. Solamente 3 cooperativas de enseñanza cerraron sus cuentas anuales a 31 de Diciembre del 2011. Dado el escaso número de cuentas anuales válidas de cooperativas de enseñanza obtenidas, se optó por su incorporación al estudio sin el objetivo de realizar un análisis separado de las mismas.

Paralelamente, se decidió incorporar al estudio el caso de Eroski, S.Coop. Al tratarse de una cooperativa de consumo no está inscrita en ERKIDE, pero debido a su volumen y la mayor diversidad de instrumentos de financiación emitidos, resulta una referencia de interés en cualquier estudio del movimiento cooperativo.

De este modo, la muestra final de cooperativas estudiadas está compuesta por 72 empresas de las que 65 presentan cuentas anuales auditadas (las otras 7 empresas, estaban exentas de obligación de auditoría de cuentas). Por otro lado, el 58\% de las cooperativas (47 empresas) pertenecen al grupo Cooperativo Mondragón.

Tabla 2: Tipos de cooperativa de la muestra final

\begin{tabular}{lc}
\hline \multicolumn{2}{c}{ Cooperativas analizadas } \\
\hline Cooperativas de Trabajo Asociado & 68 \\
Cooperativas de Enseñanza & 3 \\
Eroski, S.Coop & 1 \\
\hline Número total de cooperativas estudiadas & $\mathbf{7 2}$
\end{tabular}

Fuente: Elaboración propia 
De acuerdo con el régimen transitorio previsto en la disposición transitoria única de la Orden EHA/3360/2010, en el primer ejercicio en que se apliquen las normas, en la medida que deban reclasificar determinadas partidas de patrimonio al pasivo de la entidad, lo harán por el valor contable que presenten las mismas al inicio de dicho ejercicio. Por otra parte, no se rectificarán los importes que se hubieran contabilizado de acuerdo con la normativa vigente en el ejercicio anterior a la primera aplicación de la misma, por lo que los valores contenidos al cierre de dicho ejercicio se considerarán valores iniciales a los efectos de la nueva norma.

La interpretación de dicha disposición transitoria por parte de las empresas estudiadas ha resultado heterogénea, dificultando la aplicación de nuestro estudio. En torno a un $20 \%$ de las empresas analizadas ha presentado la cuenta de pérdidas y ganancias del 2010 con la estructura prevista en la antigua Orden ECO/3614/2003, definiendo la variable de excedente de la cooperativa después de la determinación del resultado del ejercicio. Este hecho dificultaba un análisis comparado entre ambos ejercicios.

Por otro lado, la mayoría de las cooperativas ha optado por facilitar la comparabilidad de la información de pérdidas y ganancias presentando los resultados del 2010 bajo la estructura de la cuenta de resultados aprobada por la Orden EHA/3360/2010. No obstante, este esfuerzo de adaptación ha ofrecido resultados poco satisfactorios en lo que respecta a la coherencia de la reclasificación de los intereses retribuidos al capital en el 2010, ya que en ocasiones eran reclasificados como aplicación del resultado, mientras que en otros casos se seguían presentando como gasto dentro del resultado financiero. Esta circunstancia ha exigido un esfuerzo de homogenización y reelaboración de la información presentada en los estados financieros de las diferentes cooperativas especialmente al cierre 2010, a fin de obtener unos resultados consistentes. La reclasificación de la dotación del fondo de educación como componente del resultado de explotación ha resultado más acertada en la mayoría de los casos.

\section{RESULTADOS}

\subsection{Medidas acordadas por las cooperativas a fin de proteger el Patrimonio Neto}

Como primer objetivo del trabajo, estudiamos la información cuantitativa y cualitativa ofrecida en las cuentas anuales con relación a la consideración del capital social y otras partidas de fondos propios con motivo de la entrada en vigor de la nueva normativa contable así como las posibles repercusiones que ello haya conllevado. De las 72 empresas analizadas, 
encontramos que en 5 casos (6,9\%) no se ha aplicado la nueva Orden EHA/3360/2010. Resulta especialmente significativo el caso de una cooperativa que casualmente inicia el ejercicio el 31 de diciembre del 2010 y cierra a 30 de diciembre del 2011, suponemos que en un intento de retrasar la aplicación de la norma.

Entre las empresas en las que encontramos indicios de que hayan aplicado la nueva normativa, la opción mayoritaria es aquélla en la que los socios han tomado la decisión de renunciar a favor del Consejo Rector o la Asamblea General el derecho a rehusar incondicionalmente el reembolso. Esta circunstancia, que se ha producido en 38 cooperativas (52,7\% de la muestra final total), ha permitido que sigan contabilizando el capital social de modo íntegro como parte de los Fondos Propios.

En algunas de estas memorias hemos encontrado justificaciones en torno al principio general que ha de presidir la regulación de la disponibilidad en caso de baja de las aportaciones, que reside en la necesidad de evitar la descapitalización de la cooperativa en cuantías inaceptables para su continuidad como empresa, y en todo caso, asegurar el carácter de capital y de los fondos propios de las aportaciones de los socios. Las fechas en las que las asambleas han tomado la decisión de rehusar al mencionado derecho, abarcan desde 2006, en el caso más prematuro, hasta el propio ejercicio 2011. Es también frecuente hacer referencia a las medidas cautelares recogidas en la propia legislación de cooperativas, señalando que mientras se mantenga alguna aportación cuyo reembolso hubiese sido rehusado por decisión del Consejo Rector, los órganos sociales de la cooperativa no podrán adoptar ningún acuerdo de devengo de intereses de las aportaciones, ni acuerdo de distribución de retornos, y que en el Reglamento Interno se regulará la retribución que corresponde, en su caso, a las aportaciones cuyo reembolso hubiera sido rehusado.

Otra de las soluciones adoptadas por las cooperativas analizadas ha consistido en la renuncia y/o reclasificación parcial en base a diferentes criterios.

- A raíz de la consulta del ICAC (2011), alguna cooperativa ha optado por ceder a favor del Consejo Rector el derecho a rehusar incondicionalmente el reembolso, con la excepción de los casos de jubilación o incapacidad, en cuyo caso se mantendría dicho derecho. Esta renuncia, menos agresiva y lesiva para los intereses y derechos tradicionales de los cooperativistas, resulta compatible con el mantenimiento del 100 por cien del capital como Fondo propio en las cooperativas de trabajo asociado. A 
este respecto, otras cooperativas han ido más allá en su interpretación, puesto que al tratarse de cooperativas de trabajo asociado y dado que las opciones de baja de socios más probables se relacionan con procesos de jubilación o incapacidad, contemplados en la consulta del ICAC, consideran que no es necesario renunciar al derecho de rescate para mantener el capital como fondo propio.

- Otra de las soluciones planteadas consiste en limitar anualmente en Asamblea General el importe máximo de los reembolsos, de forma que el exceso puede ser rehusado. Esta solución ha sido planteada en 8 de las cooperativas analizadas (11,1\% de la muestra). Las limitaciones establecidas abarcan distintas posibilidades, desde cuantías fijas aprobadas anualmente en Asamblea General, hasta el establecimiento de porcentajes fijos sobre el capital social. La solución planteada más habitual consiste en limitar anualmente en Asamblea la cantidad reembolsable máxima que no requiera el acuerdo del Consejo Rector. Este acuerdo tendría vigencia anual hasta la celebración de la siguiente Asamblea que podría ir modificando y adaptando dicha cuantía. En algún caso, en el acuerdo se han establecido límites porcentuales sobre el capital en términos genéricos, pero no nos consta que estén referidos o sujetos a un importe fijo. Se da la circunstancia de que sólo dos cooperativas han reclasificado como pasivo la parte del capital en la que el Consejo Rector carece del derecho inexcusable de rechazar su reembolso.

Vargas (2011) ya había identificado la ambigüedad con la que muchas de las reformas legislativas de las diferentes comunidades autónomas han tratado la posibilidad de limitar la devolución a un determinado porcentaje de aportaciones de capital. Posteriormente, una consulta del Instituto de Contabilidad exige vincular el porcentaje establecido a una cifra fija de capital a fin de poder mantener el capital que excede dicho porcentaje como fondo propio (ICAC, 2013). A nuestro entender, según la información disponible que hemos podido analizar, la mayoría de las soluciones planteadas en los casos estudiados no cumplirían el requisito exigido por la consulta $\mathrm{y}$, por tanto, deberían ser presentados como pasivos según la interpretación del ICAC (2013).

- Otra de las soluciones ha consistido en la renuncia de socios al derecho de rescate, únicamente por la parte de las aportaciones obligatorias que han podido mantenerse 
en los fondos propios. El resto de aportaciones voluntarias de capital se han tenido que reclasificar como pasivo.

- En varias empresas la reclasificación del capital ha estado vinculada a los diferentes compromisos de remuneración asociados a diferentes formas de capital. Por ejemplo, en una cooperativa de educación, el capital de los socios colaboradores se remunera a un porcentaje respecto del interés legal del dinero, por lo que esa parte del capital ha sido reclasificada como pasivo, pero el capital de los socios usuarios no se remunera, y el Consejo Rector queda facultado a rehusar incondicionalmente cualquier solicitud de reembolso. En el caso de Eroski, las obligaciones acordadas de remuneración han obligado a la reclasificación como pasivo de parte de las emisiones de aportaciones financieras subordinadas.

El caso de reclasificación de capital más extremo se ha producido en una cooperativa de educación cuya asamblea de socios, a fecha de cierre del ejercicio, tiene aún pendiente de adoptar el acuerdo referente a la facultad de rehusar el reembolso del capital social, por lo que el total de la cifra de capital social ha sido íntegramente reclasificada al pasivo no corriente.

Finalmente, hay 11 empresas (15,28\% de la muestra) que, recogiendo en sus cuentas anuales la entrada en vigor de la nueva norma sobre contabilidad de cooperativas, mantienen el capital en el neto sin ofrecer ninguna explicación ni detalle sobre las posibles decisiones que hayan tomado.

Tabla 3: Opciones acordadas sobre la consideración del capital en aplicación de la nueva normativa

\begin{tabular}{|c|c|c|c|}
\hline Decisiones de la empresa & $\begin{array}{l}\text { Número de } \\
\text { empresas }\end{array}$ & Porcentaje & \\
\hline Renuncia incondicional al derecho de reembolso & 38 & $52,78 \%$ & \\
\hline Limita el importe máximo anual de reembolsos & 8 & $11,11 \%$ & \\
\hline $\begin{array}{l}\text { Renuncia en la parte que corresponde a algún tipo de } \\
\text { aportaciones }\end{array}$ & 5 & $6,94 \%$ & \\
\hline $\begin{array}{l}\text { Renuncia al derecho a reembolso excepto en caso de } \\
\text { jubilación o incapacidad }\end{array}$ & 1 & $1,39 \%$ & $53=73,6 \%$ \\
\hline $\begin{array}{l}\text { No ha ratificado la decisión de renuncia pero está } \\
\text { previsto hacerlo }\end{array}$ & 1 & $1,39 \%$ & \\
\hline $\begin{array}{l}\text { No renuncian al derecho a rescate (acogiéndose a la } \\
\text { consulta del ICAC o no) }\end{array}$ & 3 & $4,17 \%$ & \\
\hline
\end{tabular}




\begin{tabular}{lcc}
$\begin{array}{l}\text { Aplica la Orden EHA/3360/2010 pero no aporta } \\
\text { explicaciones sobre consideración de capital } \\
\text { relacionadas con ella }\end{array}$ & 11 & $15,28 \%$ \\
$\begin{array}{l}\text { No aplica la Orden EHA/3360/2010 } \\
\text { Total }\end{array}$ & 5 & $6,94 \%$ \\
\hline
\end{tabular}

Fuente: Elaboración propia

Resumiendo los resultados obtenidos, el 73,6\% de las cooperativas estudiadas han tomado alguna decisión estatutaria que implica algún tipo de renuncia de sus derechos tradicionales a fin de poder mantener total o parcialmente el capital como fondo propio. Tan solo en 3 casos, en aplicación extensiva de la consulta del ICAC (2011) la condición de cooperativas de trabajo asociado ha permitido no realizar ninguna renuncia sobre los derechos de reembolso. En el resto de los casos (22,22\% de la muestra) no se aportan explicaciones sobre las decisiones adoptadas, o bien, no hemos encontrado ningún indicio de la aplicación de la nueva norma contable.

\subsection{Impacto cuantitativo sobre variables del Patrimonio Neto}

Como segundo objetivo de nuestro trabajo, hemos analizado los efectos que la aplicación de la nueva norma contable haya podido suponer sobre las principales variables de fondos propios y los correspondientes ratios de análisis de solvencia y posición financiera. Como hemos señalado anteriormente, la mayor parte de las cooperativas analizadas han evitado los aspectos más polémicos de la incidencia de la aplicación de la nueva norma contable, cediendo a favor de la Asamblea General o el Consejo Rector, el derecho incondicional a rehusar el reembolso de las solicitudes de capital. Sin embargo, las cooperativas que no han renunciado a dicho derecho de modo íntegro han sufrido importantes consecuencias sobre las partidas de capital y reservas.

Según los resultados de nuestro estudio, son 8 las empresas que han reclasificado alguna de las partidas de fondos propios como consecuencia de la entrada en vigor de la nueva norma. En términos cuantitativos, son especialmente relevantes 4 casos de cooperativas que han reclasificado parte de su capital. Hay un único caso en el que la cooperativa ha reclasificado el $100 \%$ de su capital como pasivo, cuya memoria señala la transitoriedad del ajuste de reclasificación, indicando que está previsto aprobar en futuras asambleas la cesión a favor del Consejo Rector, del derecho incondicional a rehusar el rescate. En otros dos casos, 
se ha reclasificado el capital original como pasivo, manteniéndose únicamente como capital las aportaciones obligatorias. En estos dos casos, el ajuste ha supuesto la pérdida del $96,5 \%$ y $64,5 \%$ del capital original respectivamente. En el cuarto caso la cooperativa ha reclasificado como pasivo el capital aportado por los socios colaboradores, argumentando la existencia de un derecho de remuneración no discrecional, y el capital reclasificado ha supuesto en $44 \%$ del capital total de la cooperativa. En el resto de cooperativas, el capital reclasificado corresponde al importe que la Asamblea General autoriza reembolsar como máximo a lo largo del ejercicio siguiente, que lógicamente supone un porción menos relevante, menor al 2\% del capital original en ambos casos.

Son, a su vez, dos los casos en los que las cooperativas reclasifican reservas voluntarias como consecuencia de la aplicación de la nueva norma. Una cooperativa en la que sus socios no han renunciado al derecho de rescate se ve obligada a reclasificar las reservas voluntarias repartibles, ajustando el $54,2 \%$ de la partida original. En el otro caso el ajuste es poco relevante, menor al $1 \%$ de la partida, si bien su origen no está detallado en las cuentas correspondientes.

Finalmente Eroski se vio obligada a reclasificar como pasivo el 54,4\% de sus aportaciones financieras subordinadas. En este caso, el principal obstáculo para su mantenimiento como fondo propio surgía de los compromisos adquiridos de remuneración obligatoria, dado que la cooperativa no tiene asumida ninguna obligación de reembolso hasta la liquidación de la misma.

Tabla 4: Ajustes más relevantes sobre partidas de fondos propios

\begin{tabular}{|l|c|c|c|c|c|c|}
\hline \multirow{2}{*}{ RECLASIFICACIONES } & \multirow{2}{*}{$\begin{array}{c}\text { No de } \\
\text { Coop. }\end{array}$} & \multicolumn{5}{|c|}{$\%$ sobre volumen previo de la partida (cierre 2010) } \\
\cline { 3 - 7 } & & $0 \%-19 \%$ & $20 \%-39 \%$ & $40 \%-59 \%$ & $60 \%-79 \%$ & $80 \%-100 \%$ \\
\hline Capital social & 6 & 2 & & 1 & 1 & 2 \\
\hline Reservas & 2 & 1 & & & 1 & \\
\hline Fondos capitalizados & 1 & & & 1 & & \\
\hline
\end{tabular}

Fuente: Elaboración Propia

Si analizamos el volumen acumulado de reclasificaciones sobre el conjunto de las partidas de fondos propios, destaca un caso en el que la cooperativa al acumularse los ajustes de capital y reservas voluntarias pierde $61,5 \%$ de sus fondos propios. En el resto de los ejemplos, los ajustes totales acumulados oscilan entre el $30 \%$ y el $20 \%$ de los fondos propios, 
mientras que en tres casos, el ajuste resulta claramente residual, afectando a menos del $1 \%$ del total de los fondos propios.

Tabla 5: Ajustes Acumulados sobre el total Fondos propios

\begin{tabular}{|c|c|c|c|c|c|}
\hline \multirow{2}{*}{} & \multicolumn{5}{|c|}{$\%$ Reclasificado sobre volumen total de Fondos propios (cierre 2010) } \\
\cline { 2 - 5 } & $0 \%-19 \%$ & $20 \%-39 \%$ & $40 \%-59 \%$ & $60 \%-79 \%$ & $80 \%-100 \%$ \\
\hline $\begin{array}{c}\text { Número de } \\
\text { cooperativas }\end{array}$ & 3 & 4 & & 1 & 0 \\
\hline
\end{tabular}

Fuente:Elaboración Propia

A fin de evaluar el impacto sobre el ratio de solvencia global (Activo Total/Pasivo Exigible), en las empresas afectadas por reclasificaciones se han calculado dos ratios de solvencia global al cierre del 2010 (tabla 6). En el primer caso, "Solvencia global previa", se presentan los índices elaborados en base a datos contables antes de la aplicación de nueva norma. Por su parte, el ratio de "Solvencia global ajustada" incorpora, a la misma fecha, los efectos de las reclasificaciones realizadas como consecuencia de la entrada de la nueva normativa. Tal y como se ha señalado anteriormente, los planteamientos heterogéneos de presentación de la información comparativa del 2010 por parte de las empresas analizadas, han requerido una labor de homogenización de la información presentada en las cuentas anuales de dicho ejercicio. Para ello, sobre los datos previamente homogeneizados de cierre del 2010, se han ajustado las reclasificaciones que ha realizado cada cooperativa por efecto de la nueva normativa.

Tabla 6: Efecto sobre el ratio de solvencia global en las cooperativas que han reclasificado partidas de Fondos Propios

\begin{tabular}{|l|c|c|c|c|c|c|c|c|}
\hline \multicolumn{1}{|c|}{ Cierre 2010 } & \multicolumn{7}{|c|}{ Cooperativas afectadas por reclasificaciones de fondos propios } \\
\hline & 1 & 2 & 3 & 4 & 5 & 6 & 7 & 8 \\
\hline $\begin{array}{l}\text { Solvencia Global } \\
\text { Previa }\end{array}$ & 1,81 & 1,96 & 3,60 & 4,82 & 1,54 & 2,17 & 2,08 & 6,53 \\
\hline $\begin{array}{l}\text { Solvencia Global } \\
\text { Ajustada }\end{array}$ & 1,80 & 1,94 & 3,53 & 4,70 & 1,39 & 1,77 & 1,25 & 2.52 \\
\hline $\begin{array}{l}\text { \% de pérdida en } \\
\text { solvencia global }\end{array}$ & $0,24 \%$ & $1,16 \%$ & $1,97 \%$ & $2,40 \%$ & $9,74 \%$ & $18,54 \%$ & $40,12 \%$ & $61,46 \%$ \\
\hline
\end{tabular}

Fuente:Elaboración Propia 
Como era de esperar, las empresas con importantes ajustes ven reducidos los ratios de solvencia de modo significativo, destacando dos casos en los que la reducción acumula un $61,46 \%$ y un $40,12 \%$ respectivamente.

Por último, es necesario señalar el efecto indirecto positivo que la entrada en vigor de la Orden EHA/3360/2010 produce sobre el volumen del patrimonio neto al cierre del ejercicio, a través de la variable del resultado de la cooperativa. En concreto, es consecuencia del tratamiento de los intereses al capital, considerados como partida minoradora del excedente de la cooperativa según la normativa previa, y que bajo la nueva norma, ante la consideración de fondo propio del capital, se han de tratar como aplicación del resultado. Si el capital cumple los requisitos para su presentación como fondo propio, según la nueva norma contable, los intereses pagados no restan el saldo del resultado, y por tanto, se produce un aumento del resultado acumulado que las cuentas anules ofrecen en los fondos propios. Este hecho, ha supuesto un efecto positivo sobre el patrimonio neto de las cooperativas que hayan conseguido mantener el capital como neto.

Tal como hemos comentado, según los datos de nuestro estudio, y fruto de diversas decisiones tomadas en sus asambleas la mayor parte de las cooperativas analizadas han seguido manteniendo el capital como elemento integrante de los fondos propios. Bajo esta hipótesis, si la cooperativa ha pagado intereses al capital, la aplicación de la nueva norma implicará recalcular la variable del excedente, dejando de restar el importe de los intereses como partida de gasto. Esta circunstancia aplicada sobre los valores de cierre de ejercicio 2010, impondría un aumento del importe de los fondos propios en 49 (el 68\%) de las cooperativas analizadas.

Tabla 7: Ajuste indirecto sobre Fondos Propios por reclasificación del interés pagado al capital

\begin{tabular}{|c|c|c|c|c|c|c|}
\hline & \multicolumn{6}{|c|}{$\%$ de aumento del total de fondo propio (cierre del 2010) } \\
\cline { 2 - 7 } & $0 \%-1 \%$ & $1 \%-2 \%$ & $2 \%-3 \%$ & $3 \%-4 \%$ & $4 \%-5 \%$ & $5 \%-6 \%$ \\
\hline $\begin{array}{c}\mathrm{N}^{\mathrm{o}} \mathrm{de} \\
\text { empresas }\end{array}$ & 7 & 7 & 11 & 11 & 12 & 1 \\
\hline
\end{tabular}

Fuente:Elaboración Propia

Porcentualmente, en el caso más extremo el impacto sobre los fondos propios supone un aumento del 5,8\% del total de fondos propios. En los casos más frecuentes (34 cooperativas), el efecto oscila entre un $2 \%$ y un $5 \%$ del total de los fondos propios. En todo caso, hay que recordar que este aumento de los fondos propios es transitorio, es decir, que no 
se consolida, dado que, si se pagan, tras la distribución de resultados desaparecerán del neto de la cooperativa.

\section{DISCUSIÓN DE RESULTADOS}

Entre las diferentes fórmulas que las cooperativas han aprobado para protegerse del eventual impacto negativo de la aplicación del nuevo marco normativo contable destaca, en el $74 \%$ de las cuentas analizadas, la opción de ceder a favor del Consejo Rector el derecho a rehusar incondicionalmente el rescate del capital y otros fondos recuperables. Esta cesión se ha producido de modo pleno, 53\% de los casos, o sujeta a diversas limitaciones, por tipo de aportaciones, casos de jubilación o incapacidad, etc. que sumarían el $21 \%$ restante. Únicamente 3 cooperativas de trabajo asociado (4,17\%), mantienen el capital como neto sin realizar ninguna renuncia, basando dos de ellas su argumento en la consulta publicada por el ICAC (2011).

A nuestro entender, la fecha tardía en la que se publicó dicha consulta, hizo que la mayoría de la cooperativas tomasen, previamente, la decisión de proteger sus intereses patrimoniales, cediendo parte de sus derechos tradicionales, y evitando así la amenaza que implicaba la reclasificación de las variables centrales del patrimonio neto tradicional de las cooperativas como pasivos. Es, a nuestro juicio, una interpretación valiente, acertada y en línea con algunas decisiones provisionales adoptadas en el marco de los organismos internacionales de contabilidad. Sin embargo, dicha reflexión resulta de difícil encaje con el texto íntegro de la Orden EHA 3360/2010. No cabe duda de que una referencia interpretativa explícita en la norma al hecho diferencial del caso de las cooperativas de trabajo asociado, o los casos de jubilación e incapacidad o la circunstancia expresa de incapacidad de seguir desarrollando la actividad tras el reembolso habría evitado, o al menos modificado, la mayoría de las decisiones adoptadas en el seno de las asambleas de las cooperativas. A este respecto son reveladores algunos casos en los que en Asamblea General se ha acordado la cesión a favor del Consejo Rector del derecho incondicional a rehusar el rescate excepto en los casos de jubilación o incapacidad.

En buena parte de los casos analizados (15\%), a pesar de que las cooperativas mantenían el capital como fondo propio, las cuentas anuales no aportan información sobre la justificación de dicha decisión. El hecho de que únicamente hayamos analizado la información contenida en la Memoria de las cuentas anuales supone una limitación, pudiendo 
resultar que los Estatutos y Reglamentos de funcionamiento interno contengan más detalles de información que los aquí recogidos. Una extensión de esta investigación podría consistir en estudiar la muestra para el ejercicio 2012, a fin de comprobar si se mantiene la tendencia de renuncia o si las empresas se acogen en mayor grado a la consulta del ICAC (2011), incluso tomando decisiones asamblearias de recuperación del derecho al que hayan renunciado.

Con relación al análisis de los efectos cuantitativos, segundo de los objetivos, los resultados de la investigación muestran, en primer lugar, que la aplicación del cambio normativo no ha tenido un impacto relevante sobre los fondos propios de la mayoría de las cooperativas de trabajo asociado de mayor número de empleados de Euskadi en el ejercicio 2011. Tan sólo 8 de las 72 cooperativas estudiadas (11\%) ha reclasificado alguna partida de capital, reservas o fondos capitalizados. De este modo, el efecto conjunto de los ajustes realizados no ha sido significativo sobre la muestra analizada. Los ajustes aplicados sobre el capital social han supuesto una disminución del $0,379 \%$ del capital cooperativo acumulado por las cooperativas estudiadas. En lo que respecta a las reservas, la disminución ha sido del $0,074 \%$ de reservas acumuladas por las cooperativas de la muestra.

Este efecto global poco significativo no ha de ocultar un impacto patrimonial, proporcionalmente relevante, sobre las cooperativas que se han visto forzadas a realizar reclasificaciones. En cinco de las cooperativas estudiadas se ha perdido más del $20 \%$ de sus fondos propios, alcanzando un ajuste extremo del 61,5\% de los fondos propios en uno de los casos. Se da la circunstancia de que estas cinco empresas dos son cooperativas de enseñanza. Sin embargo, la estrechez de la muestra del colectivo de enseñanza no permite establecer ninguna relación significativa a este respecto.

El único ajuste global significativo $(52,83 \%)$ se ha producido sobre la partida de los fondos capitalizados u otros instrumentos de patrimonio neto motivado, exclusivamente, por la reclasificación como pasivo de las primeras emisiones de aportaciones subordinadas de Eroski, S.Coop debido a los compromisos obligatorios de remuneración asumidos en la emisión. Se trata, sin duda alguna, de un modelo de financiación menos enraizado en el movimiento cooperativo, cuya clasificación como pasivo es coherente y menos lesiva sobre los principios cooperativos tradicionales. Por otra parte, solamente una de ellas se ha visto obligada a reclasificar parte de estos instrumentos. 
Finalmente, en las cooperativas que han logrado mantener el capital como elemento integrante de los fondos propios y, a su vez, han remunerado con interés dichas aportaciones, la aplicación de la nueva norma ha supuesto considerar dicha remuneración como aplicación de resultado. Esta circunstancia tiene una incidencia notable sobre el volumen del resultado del ejercicio presentado en los fondos propios. Además, de modo indirecto, supone un aumento del importe de los fondos propios. En nuestro estudio este hecho ha supuesto, en el $68 \%$ de las empresas analizadas, un incremento de medio de los fondos propios del 2,8\%. Este incremento del neto es, sin embargo, transitorio hasta aprobación y ejecución de la propuesta de aplicación del resultado, a no ser que la Asamblea de socios opte por la capitalización de los mismos.

\section{CONCLUSIONES}

La aplicación del cambio normativo no ha tenido un impacto relevante sobre los fondos propios de la mayoría de las cooperativas de trabajo asociado con más de 50 empleados de Euskadi, fundamentalmente, porque las cooperativas han optado por cambiar sus condiciones estatutarias a fin de protegerse del efecto de la aplicación del nuevo marco normativo contable. Sin embargo, hemos constatado que las cooperativas que no han adaptado su marco estatutario a las exigencias de la nueva regulación se han visto forzadas a realizar reclasificaciones de fondos propios a pasivo, que han tenido un impacto patrimonial muy relevante.

Los resultados obtenidos en nuestro estudio difieren de los obtenidos en otros trabajos que han analizado el mismo fenómeno (Bastida, Amat y Campa, 2013) en los que el efecto conjunto de los ajustes se ha mostrado significativo. El mayor esfuerzo de adaptación de Estatutos de las cooperativas del País Vasco parece vislumbrarse como el factor determinante de la diferencia de resultados obtenidos. A pesar de que ambos estudios se han realizado sobre cooperativas medianas y grandes, las diferencias en la tipología de cooperativas estudiadas, las diferencias geográficas o la pertenencia o no a organizaciones empresariales de coordinación como Mondragón Corporación Cooperativa deberán ser consideradas en futuros trabajos como factores que ayuden a explicar los diferentes comportamientos.

La regulación contable ha sido tradicionalmente desarrollada pensando en su aplicación en las sociedades de capital. El encaje de dicha normativa en entornos diferenciados, como el caso de las cooperativas, genera consecuencias que pueden ocasionar 
la modificación de parámetros clásicos de la relación de la cooperativa con sus socios. A nuestro juicio, persistiendo en algunas de las decisiones provisionales adoptadas, los reguladores contables deberían considerar en mayor medida el impacto de los cambios normativos sobre la información publicada por las empresas, en realidades y tipologías de negocios diferentes a las sociedades de capital, como es el caso las cooperativas.

La definición de los elementos de neto y pasivo, así como la fijación de unos criterios consistentes de distinción entre instrumentos financieros de neto y pasivo, es una cuestión inacabada, que ahora parece activarse nuevamente en el foro de la discusión sobre el marco conceptual del IASB. En cualquier caso, en este debate, el caso de las cooperativas ha sido propuesto en repetidas ocasiones como ejemplo donde la normativa actual requiere un nuevo recorrido a fin de resolver algunos de los efectos no deseados. En este sentido, nos parece importante la receptividad mostrada por el organismo regulador internacional IASB para incluir en el marco conceptual características de los elementos de pasivo y neto, que permitan en el futuro crear excepciones para responder a las especificidades manteniendo la coherencia del modelo.

El regulador español ha incorporado algunas de las propuestas de este debate a través de consultas que, a nuestro juicio, son difíciles de inferir desde el texto íntegro de la Orden EHA 3360/2010. Una referencia interpretativa explícita en la norma al hecho diferencial del caso de las cooperativas de trabajo asociado, o los casos de jubilación e incapacidad o la circunstancia expresa de incapacidad de seguir desarrollando la actividad tras el reembolso habría evitado, o al menos modificado, la mayoría de las decisiones adoptadas en el seno de las asambleas de las cooperativas.

\section{BIBLIOGRÁFÍA}

ANDICOECHEA ARONDO, L.; ZUBIAURRE ARTOLA, M.A. (2012) Equity-Liability Accounting Debate in Worker Co-operative Entities Members' Shares. Journal of Cooperative Accounting and Reporting, V1, N1, Summer 2012. p. 28-46. Disponible en Internet: http://www.smu.ca/webfiles/A3-JCAR-2102-1-1.pdf

ARIAS, B.; MONTEGUT, Y. (2012) Los efectos de la nueva normativa contable en las cooperativas. Revista de Contabilidad y Dirección, $n^{o}$ 14, p. 183-201. Disponible en Internet: 
http://www.accid.org/revista/documents/Los_efectos_de_la_nueva_normativa_contable_e n_las_cooperativas.pdf. ISSN: 1887-5696.

BASTIDA, R.; AMAT, O.; CAMPA F. (2013) Efectos económicos de la primera aplicación de las normas contables adaptadas a la NIC 32 en las cooperativas. 4th CIRIEC International Research Conference on Social Economy. Amberes, Bélgica.

FASB (Financial Accounting Standards Board) (2007) Financial Instruments with Characteristics of Equity. Preliminary Views. Disponible en: http://www. fasb.org

FERNÁNDEZ GUADAÑO, J. (2007) Divergencias entre las normas internacionales de información financiera y las normas sobre aspectos contables de las sociedades cooperativas: Efectos sobre la solvencia financiera. Comunicación presentada al $X X$ Congreso Anual de AEDEM Decisiones Basadas en el Conocimiento y en el Papel Social de la Empresa, p. 885-893.

IASB (International Accounting Standards Board) (2004) Aportaciones de Socios de Entidades Cooperativas e Instrumentos Similares, CINIIF2. Disponible en: http://www.ifrs.org.

IASB (International Accounting Standards Board) (2008) Financial Instruments with Characteristics of Equity. Discussion Paper. Disponible en: http://www. ifrs.org.

IASB (International Accounting Standards Board) (2010) El marco conceptual para la Información Financiera. Capítulo 1: El objetivo de la información financiera con propósito general. Capítulo 3: Características cualitativas de la información financiera útil. Septiembre. Disponible en: http://www. ifrs.org

IASB (International Accounting Standards Board) (2011) Instrumentos Financieros: Presentación, NIC 32. Disponible en: http://www. ifrs.org

IASB (International Accounting Standards Board) (2013) Elements of financial statements: definition of equity and distinction between liabilities and equity instruments. Agenda ref10E(a). Disponible en: http://www. ifrs.org.

ICAC (Instituto de Contabilidad y Auditoría de Cuentas) (2011) Calificación contable de las aportaciones de los socios al capital de una sociedad cooperativa exigibles única y exclusivamente en el caso de baja obligatoria por incapacidad o jubilación. Consulta 7 , BOICAC 87, Septiembre. Disponible en Internet: http://www.icac.meh.es/Consultas/Boicac/ficha.aspx?hid=424

ICAC (Instituto de Contabilidad y Auditoría de Cuentas) (2013) Clasificación del capital social como pasivo cuando el reembolso de un porcentaje del capital está condicionado al 
acuerdo favorable del Consejo Rector. Consulta 6, BOICAC 94, Junio. Disponible en Internet: http://www.icac.meh.es/Consultas/Boicac/ficha.aspx?hid=469

LÓPEZ-ESPINOSA, G.; MADDOCKS, J.; POLO-GARRIDO, F. (2012) Co-operatives and the Equity-Liabilities Puzzle: Concerns for Accounting Standard-Setters, Accounting Horizons, Vol. 26, dec., $\mathrm{N}^{\mathrm{o}}$ 4, p. 767-787. http://dx.doi.org/10.2308/acch-50208. Disponible en Internet: http://aaajournals.org/doi/pdf/10.2308/acch-50208

MARÍ VIDAL, S. (2006) Efectos de la aplicación de la CINIIF 2 en las cooperativas. un estudio empírico en dos cooperativas citrícolas de la comunidad valenciana a través del análisis económico-financiero. REVESCO, Revista de Estudios Cooperativos, No 89, p. 84-107. Disponible en Internet: http://pendientedemigracion.ucm.es/info/revesco/txt/REVESCO\%20N\%2089.3\%20Sergio \%20MARI\%20VIDAL.pdf. ISSN: 1885-8031.

VARGAS VASSEROT, C. (2007) Los previsibles efectos de la NIC 32 en el sector cooperativo. REVESCO, Revista de Estudios Cooperativos, Nº 91, p. 120-159. Disponible en

Internet:

http://pendientedemigracion.ucm.es/info/revesco/txt/REVESCO\%20N\%2091.5\%20Carlos \%20VARGAS\%20VASSEROT.htm. ISSN. 1885-8031

VARGAS VASSEROT, C. (2011) Aportaciones exigibles o no exigibles: Ésa es la cuestión. CIRIEC-España.Revista Jurídica De Economía Social y Cooperativa, № 22, p. 75-119. ISSN 1577-4430.

\section{Legislación:}

Ley 4/1993 de 24 de Junio, de Cooperativas de Euskadi, BOPV de 19 de Julio.

Ley 27/1999 de 16 de Julio de Cooperativas, BOE nº 170, de 17 de Julio.

Ley Foral 14/2006, de 11 de Diciembre de Cooperativas de Navarra. BON no 149 de 13 de Diciembre.

Ley 16/2007, de 4 de julio, de Reforma y Adaptación de la Legislación Mercantil en Materia Contable para su Armonización Internacional con Base en la Normativa de la Unión Europea. Orden ECO/3614/2003 de 16 de Diciembre de 2003, BOE nº 310 de 27 de Diciembre. Orden EHA/3360/2010 de 21 de Diciembre de 2010, BOE nº 316 de 29 de Diciembre. 